\title{
Molecular Interaction Studies of Natural Compounds against Coronavirus Hemagglutinin-Esterase in complex with ACE2 receptor: An In Silico Approach
}

\author{
Chirag Patel ${ }^{1}$, Dharmesh Jaiswal ${ }^{1}$, Siddhi Jani ${ }^{1}$, Daxesh Patel ${ }^{2}$, Frank Gonzalez ${ }^{2}$, and \\ Himanshu Pandya ${ }^{1}$ \\ ${ }^{1}$ Gujarat University \\ ${ }^{2}$ National Institutes of Health
}

June 17, 2020

\begin{abstract}
The COVID-19 virus emerged as a global health issue and its spike protein requires angiotensin-converting enzyme2 (ACE2) and hemagglutinin-acetylesterase (HE) glycoprotein receptor for cell entry and replication, as revealed by biophysical and structural evidence. Target inhibition can lead to therapy against the COVID-19 virus. In silico analysis was employed with plant-derived phytochemicals and synthetic drugs. The molecular docking, pharmacophore, ADMET prediction and molecular dynamics simulations techniques revealed that the Withania somnifera (ashwagandha) and Asparagus racemosus (shatavari) plants possessed various steroidal saponins and alkaloids which could potentially inhibit the COVID-19 virus targeted HE glycoprotein receptor.
\end{abstract}

\section{Abbreviations:}

COVID-19 virus: Coronavirus Disease-19 virus; ACE2; angiotensin-converting enzyme 2; HE; hemagglutininacetylesterase; SARS; severe acute respiratory syndrome; RBD; receptor binding domain; S-protein; Spikeprotein; ADMET; Adsorption; Distribution; Metabolism; Excretion; Toxicity; PDB; Protein Data Bank; 3CL5; Structure of coronavirus hemagglutinin-esterase in complex with 4,9-O-diacetyl sialic acid; YASARA; Yet Another Scientific Artificial Reality Application; Assisted Model Building with Energy Refinement; MD; Molecular Dynamics; HBA; Hydrogen bond acceptor; HBD; Hydrogen bond donor; HY; hydrophobic; RA; ring aromatic; PI; positive ionizable centers; NI; negative ionizable centers; TOPKAT; TOxicity Prediction by Komputer Assisted Technology; YAMBER; YASARA assisted model building with energy refinement; pKa; dissociation constant; TIP3P; transferable intermolecular potential 3 points; DCCM; Dynamic cross correlation matrix; MM-GBSA; Molecular mechanics generalized born surface area; MM-PBSA; Molecular mechanics Poisson-Boltzmann surface area; AMBER; assisted model building with energy refinement; RMSD; Root-mean-square deviation; RMSF; Root-mean-square fluctuation; CYP2D6;Cytochrome P450; BBB; Blood brain barrier; PSA; Polar surface area;

Molecular Interaction Studies of Natural Compounds against Coronavirus HemagglutininEsterase in complex with ACE2 receptor: AnIn Silico Approach

INTRODUCTION

Coronavirus, a SARS (Severe acute respiratory syndrome) betacoronavirus is a positive-sense, single-strand RNA virus ${ }^{1,2}$ that attacks the gastrointestinal system, heart, kidney, liver, and central nervous system and mainly lower respiratory system leading to multiple organ failure ${ }^{3}$. The first step in viral infection is the interaction of its coat spike protein with sensitive human cells ${ }^{4}$. Spike proteins are surface homotrimers 
present on the virus surface composed of an S1 forms receptor-binding domain (RBD) ${ }^{5,6}$ and S2 protease activation subunits which exists in a metastable prefusion conformation and undergo a dramatic structural rearrangement to fuse the viral membrane with the host cell membrane ${ }^{7}$. Betacoronaviruses also have a shorter spike-like protein called hemagglutinin-esterase (HE) ${ }^{8}$. It is a viral envelope glycoprotein that mediates reversible binding to O-acetylated sialic acid of the host cell membrane and helps in the attachment expressed by some strains of coronavirus ${ }^{9-11}$.

SARS-CoV-spike is the largest known virus spike protein ${ }^{12}$ SARS-CoV-spike binds to target cells through angiotensin-converting enzyme2 (ACE2) expressed on the surface of epithelial cells of lungs, intestine, kidney, and blood vessels ${ }^{13}$. SARS-CoV-spike-protein bolsters the interaction with ACE2 molecules due to diverseness in the receptor-binding domain $(\mathrm{RBD})^{14}$. After binding to the cell host, a cell protease cleaves the spike protein to release a fusion peptide which allows host-cell entry ${ }^{15}$. The genome of SARS-CoV encodes different proteins and the structural spike protein binds the ACE2 receptor along with hemagglutinin esterase, and after establishing a connection with sugar elements on cell membranes, the hemagglutinin esterase (HE) of the virus produces messenger RNA and performs replication process ${ }^{16-19}$. This structural protein represents a potential target for the inhibition of replication.

As per the Ancient Indian scriptures including Rig-Veda, Atharvaveda, and Charka Sanhita, Medicinal plants are the natural sources of various phytochemicals which have high-value alkaloids, flavonoids, phenols, chalcones, coumarins, lignans, polyketides, alkanes, alkenes, alkynes, simple aromatics, peptides, terpenes, and steroids and shows antimicrobial, antifungal and antiviral properties ${ }^{20,21}$. Plant phytochemicals are used in various viral diseases for their antiviral activity, Therefore, in silico analysis was carried out to search for potential and specific inhibitors of Coronavirus using phytochemicals and synthetic drugs.

\section{MATERIALS AND METHODS}

To obtain potential leads against coronavirus HE protein various molecular modeling techniques were employed including molecular docking, pharmacophore mapping, ADMET prediction, molecular dynamics simulations, dynamic correlation matrix and binding free energy calculations ${ }^{22}$.

\section{Data collection and curation}

One hundred fifteen phytochemicals were selected from various medicinal plants like Withania somnifera $^{23}$, Asparagus racemosus ${ }^{24}$, Zinziber officinalis ${ }^{25}$, Allium sativum ${ }^{26}$, Curcuma longa ${ }^{27}$, Adhatoda vasica ${ }^{28}$. based on their antiviral activity (Table-S1 ). Besides this, we have also performed comparative studies of these phytochemicals with 437 synthetic drugs i.e. Atazanavir (Reyataz), Darunavir (Prezista), Fosamprenavir (Lexiva), Indinavir (Crixivan), Lopinavir/Ritonavir (Kaletra), Nelfinavir (Viracept), Ritonavir (Norvir), Saquinavir (Invirase), Tipranavir (Aptivus), Atazanavir/Cobicistat (Evotaz) and Darunavir/Cobicistat (Prezcobix) (Table-S2 ) (Clercq \& Li, 2016) as potential inhibitors of the HE fusion complex $^{29}$. However, the coronavirus hemagglutinin-esterase (PDB ID:3CL5) structure was selected as a receptor which contains 4,9-O-diacetyl sialic acid as a natural inhibitor ${ }^{9}$. These receptor, phytochemicals and protease inhibitors were passed through YASARA energy minimization involving Amber03 force field and geometry optimized using the steepest gradient approach (100 iterations $)^{30}$.

\section{Docking calculation}

Molecular docking experiments were implemented using hemagglutinin-esterase (HE) protein and two datasets which were pre-processed by water removal and other parameters in YASARA 20.4.24 with AutoDock Vina algorithm ${ }^{31}$ and AMBER03 force field ${ }^{32}$. Docking validation is necessary for the conformational flexibility of a protein's binding $\operatorname{site}^{30,33}$. The 4,9-O-diacetyl sialic acid was re-docked to decipher the various interactions and binding mechanisms. After that, the two datasets were applied for docking and top-ranked compounds chosen for ADMET and MD simulations analysis.

The docking score was calculated based on:

$\Delta \mathrm{G}=\Delta \mathrm{G}_{\mathrm{vdW}}+\Delta \mathrm{G}_{\text {Hbond }}+\Delta \mathrm{G}_{\text {elec }}+\Delta \mathrm{G}_{\text {tor }}+\Delta \mathrm{G}_{\text {desol }}$ 
.......(1)

Where $\Delta \mathrm{G}_{v d W}=$ van der Waals term for docking energy; $\Delta \mathrm{G}_{H b o n d}=\mathrm{H}$ bonding term for docking energy; $\Delta \mathrm{G}_{\text {elec }}=$ electrostatic term for docking energy; $\Delta \mathrm{G}_{t o r}=$ torsional free energy term for the compound when the compound transits from unbounded to bounded state; $\Delta \mathrm{G}_{\text {desolv }}=$ desolvation term for docking energy ${ }^{30}$.

\section{Pharmacophore modeling}

Ligand-based pharmacophore models ${ }^{34}$ were generated for 4,9-O-diacetyl sialic acid and top-ranked compounds from natural as well as synthetic drugs using Accelrys Discovery Studio v20.1.0.19295. The pharmacophore hypothesis relies on the interactions formed by the protein-ligand complex which shows various features. The conventional approach has pertained with default: $\mathrm{H}$ bond acceptor (HBA) and donor (HBD), hydrophobic (HY), ring aromatic (RA) and positive-negative ionizable centers. Due to the unavailability of the decoy datasets and literature the generated pharmacophores were compared with each other for conformation generation ${ }^{35,36}$.

\section{Lipinsky's rule and ADMET prediction}

Based on Lipinski ${ }^{37}$ and Veber ${ }^{38}$ rules the drug-likeness properties (molecular weight, hydrogen bond acceptor, hydrogen bond donor, lipophilicity, topological surface area, rotatable bonds) and adsorption, distribution, metabolism, excretion and toxicity (ADMET) descriptors (human intestinal absorption (HIA), blood-brain membrane, CYP-2D6 enzyme inhibition, hepatoxicity, Ames mutagenicity, carcinogenicity, rat oral LD50, etc.) were developed using SwissADME webserver ${ }^{39,40}$ and TOPKAT module of Accelrys Discovery Studio v20.1.0.19295. This procedure leads to identify the potency of drug-like molecules ${ }^{41}$.

\section{Molecular dynamics and derivation of the dynamic cross-correlation matrix}

Molecular dynamics simulations of the top-ranked five compounds were subjected to $20 \mathrm{~ns}$ time interval using the YASARA Structure suite (academic license) ${ }^{30}$. To perform the MD simulations in YASARA software certain parameters were employed accordingly which relies on energy minimization using the steepest gradient technique (100 cycles) of the YASARA energy minimization module (YASARA Biosciences, GmbH) with YAMBER (YASARA assisted model building with energy refinement) force field ${ }^{42}$, assigned AM1BCC charges (Austin model 1 semi-empirical method with bond charge correction) and restored the hydrogen bonding network in the complex ${ }^{43}$. To simulate the complex structure acid dissociation constant (pKa) was appointed to every polar amino acid in the orthorhombic cell using the AutoSMILES method with default parameters of periodic boundary condition. The simulation cell was filled with TIP3P (transferable intermolecular potential 3 points) water model which pertained $0.997 \mathrm{~g} / 1$ density and $0.9 \%$ ion concentrated $\mathrm{NaCl}$ with Berendsen thermostat $(298 \mathrm{~K})$ and barostat (1 pressure bar $)^{44,45}$. The receptor-ligand interactions were analyzed using simulation snapshots at every 25 step time trajectory ${ }^{46}$.

The dynamic cross-correlation matrix of top-ranked five compounds was constructed through $\mathrm{C} \alpha$ backbone atoms within the $8 \AA$ cut off radius to ligand atoms. The heatmaps were generated online with the help of MORPHEUS Versatile matrix visualization and analysis software ${ }^{47}$ which shows the anticorrelation and correlation between -1 and +1 matrix values. The residue motions were calculated using:

Dynamic cross correlation matrix $\mathbf{i}_{\mathbf{j}}=\frac{\langle\mathbf{d i} \bullet \mathbf{d j}\rangle}{\sqrt{\left\langle\mathrm{di}^{2}\right\rangle\left\langle\mathrm{dj}^{2}\right\rangle}}$

.......(2)

Here, $d$ is the displacement vector between the $i$ th $\mathrm{C} \alpha$ atom of a particular residue with $j$ th $\mathrm{C} \alpha$ atom of another residue as a function of time. $<>$ denotes the time ensemble average of the displacement quantities.

\section{Binding free energy calculations}

Molecular mechanics generalized born surface area (MM-GBSA) and molecular mechanics PoissonBoltzmann surface area (MM-PBSA) are being used for the binding free energy calculations ${ }^{48-51}$. Hence, 
YASARA AMBER14 with the "single trajectory approach" was used for the top-ranked five MD simulated complexes. The following equations were applied for the calculations of binding free energy $\left(\Delta \Gamma_{\beta \nu \nu \delta}\right)$ values:

$\Delta \mathrm{G}_{\text {bind }}=\Delta \mathrm{G}_{\text {complex (minimized) }}-\left[\Delta \mathrm{G}_{\text {ligand(minimized })}+\Delta \mathrm{G}_{\text {receptor(minimized })}\right]$

.......(3)

and

$\Delta \mathrm{G}_{\mathrm{bind}}=\Delta \mathrm{G}_{\mathrm{MM}}+\Delta \mathrm{G}_{\mathrm{PB}}+\Delta \mathrm{G}_{\mathrm{SA}-\mathrm{TS}}$

........(4)

Where $\Delta_{T \Delta \Sigma}$ is the conformation entropic contribution, and $\Delta \Gamma_{M M}$ is the molecular mechanics interaction energy (electrostatic + van der Waals interaction) between protein and ligand. $\Delta \Gamma_{\Pi B}$ and $\Delta \Gamma_{\Sigma A}$ depict the polar solvation energy and the nonpolar solvation energy, respectively ${ }^{52}$.

\section{RESULTS AND DISCUSSION}

Molecular docking of coronavirus hemagglutinin-esterase with plant-derived phytochemicals and synthetic drugs

Receptor-based molecular docking was employed to identify the potential and specific inhibitors of Coronavirus. However, re-docking of 4,9-O-diacetyl sialic acid was done with the HE glycoprotein and it possessed $5.23 \mathrm{kcal} / \mathrm{mol}$ with $0.1945 \mathrm{kcal} /\left(\mathrm{mol}^{*}\right.$ Atom $)$ ] efficiency with competitive inhibition. Based on the $1.84 \AA$ RMSD value it was confirmed that the re-docking of co-crystal ligand was performed successfully.Figure-1 depicts the three- and two-dimensional poses of co-crystal ligand and HE glycoprotein, in which, 3 hydrogen bonds were formed with Thr 114, Ser 116 and Tyr 184, while 2 hydrophobic interactions were also formed Phe 211 and Leu 267, this moiety plays a pivotal role in the HE glycoprotein inhibition. So, 115 plant-derived phytochemicals and synthetic drugs were docked with the coronavirus hemagglutinin-esterase complex. Among 115 plant-derived phytochemicals, Withanoside $\mathrm{V}$ was found as a potent binder with 7.8 $\mathrm{kcal} / \mathrm{mol}$ which possessed 9 hydrogen bonds with Ser 116, The 159, Tyr 184, Leu 212, Ser 213, Asn 214 and Thr 243 which resembled with the co-crystal ligand docking. The top-ranked compounds were listed for further studies. Their binding energies ranged from $7.8 \mathrm{kcal} / \mathrm{mol}$ to $7.58 \mathrm{kcal} / \mathrm{mol}$.Table-1 contains the list of top-ranked compounds having the binding energy, hydrogen bonds, the dissociation constant, efficiency (related to competitive inhibition) and contacting amino acid residues.

The synthetic drugs were also tested as a comparative study and Remdesivir bound with the highest binding energy values of $7.50 \mathrm{kcal} / \mathrm{mol}$ which was higher than the co-crystal ligand. However, Saquinavir, Delavirdine, Indinavir and Fosamprenavir were the most promising inhibitors for the HE glycoprotein (Figure-3 ). Table-2shows detailed information regarding docking scores and other details. Based on the docked scores of Withanoside $\mathrm{V}$ and Remdesivir was the robust binder of more than $7 \mathrm{kcal} / \mathrm{mol}$ binding energy. Thus, these two could be employed for HE glycoprotein inhibition after the experimental validation

\section{Pharmacophore modeling}

Ligand-based pharmacophore modeling was carried out for co-crystal ligand and top-ranked compounds due to the lack of the decoy datasets for coronavirus target and the generated pharmacophore scores were considered for the hypothesis generation from natural as well as synthetic drugs. A total of 11 pharmacophore features were generated through Accelrys Discovery Studio which possessed 4-H bond acceptor (HBA) and 6-donor (HBD) and 1-negative ionizable center. Based on this hypothesis, two separate pharmacophores were developed from top-ranked plant-derived phytochemicals and synthetic drugs and compared with the developed hypothesis. The fit scores were used to decipher the pharmacophores reliability. However, phytochemicals and proteases were notified with 2 which was more accountable for the development of developing receptor-ligand interactions. Figure- $\mathbf{4}$ demonstrates the comparative pharmacophores generated from the co-crystal ligand and top-ranked phytochemicals as well as protease inhibitors. These comparative pharmacophore modelling showed higher number of pharmacophore features development concerning co-crystal 
ligand. Hence, these hypotheses indicate the potency towards the molecular level interactions with selected HE glycoprotein targets.

\section{Lipinsky's rule and ADMET prediction}

The Lipinsky's rule of five and ADMET prediction of top-ranked compounds were studied to understand the amenability of pharmacokinetic properties and toxicity properties. Table-3 and Table- 4 depict the Lipinski's rule-of-five and ADMET prediction of top-ranked phytochemicals and FDA-approved protease inhibitors. All phytochemicals were showed violations in Druglikeness and ADMET PSA 2D properties, while ADMET Solubility Level, ADMET BBB Level, ADMET AlogP98 and $\log \mathrm{Po} / \mathrm{w}$ were successfully satisfied.

\section{Molecular dynamics simulations}

MD simulations of top-ranked 5 phytochemicals were executed for $20 \mathrm{~ns}$ time interval. The structural level of integrity and conformational changes were identified through energy, RMSD, RMSF plots of all complexes. The DCCM and binding free energy calculations were carried out also. The total energy values of all complexes were ranges from -1335000 to $-1360000 \mathrm{kcal} / \mathrm{mol}$ (Figure-5 ). However, the RMSD values have fluctuated between 1 to 4 while Shatavarin X was the only compound that possessed more variation (Figure-6 ).Figure-7 to Figure-11 depicts the changes that occurred in the conformation during the MD event. In Figure-7, the changes in the structure of HE glycoprotein and Withanoside V were notified after the $10 \mathrm{~ns}$ time trajectories which showed the decrease in the interaction generation. While Racemoside $\mathrm{C}$ was found with a dramatic change in interaction development (Figure-8). Withanoside IV depicted the fewer changes were observed in the MD event (Figure-9 ). The fluctuation was found in the Shatavarin X at every time trajectories (Figure-10 ). However, Shatavarin X pertained the fall of the interactions after 10 ns (Figure-11).

\section{Dynamic cross-correlation matrix to study residue motions of cross-terms}

The correlative motions of different pocket residues were developed from the Dynamic cross-correlation matrix. Figure-S1 to Figure-S5 . The DCCM was plotted through heatmap in the MORPHEUS online tool which provides blue and red color intense representation to decipher the residue motions of selected protein-ligand complexes. The blue color shows the negative or anti-correlated movements of pocket residues while the positive correlation in the residue motions indicated by red color. Tyr 108, Lys 112, Thr 115, Ser 116 and Thr 243 were the most common amino acid residues for the positive correlation.

\section{Binding free energy evaluation}

YASARA software was used to calculate the binding free energy of all protein-ligand complexes which included a 20 ns time interval with 200 snapshots. Figure-12 shows the MM-/PBSA calculation of all complexes, in which, Withanoside IV and Withanoside V showed the same stability with energy values. The higher energy values were observed in Racemoside $\mathrm{C}$ which ranged between -309.216 to $-20.871 \mathrm{KJ} / \mathrm{mol}$. The overall stability was noted to be Racemoside C, Shatavarin VII, Shatavarin X followed by Withanoside IV and Withanoside $\mathrm{V}$ which illustrates the partial contribution towards the inhibitory activity (Table-5 ).

\section{CONCLUSION}

The current pandemic COVID-19 virus is largely untreatable with present therapeutic drugs and no vaccine is currently available. The spike protein with HE fusion complex was targeted in silico with plant-derived phytochemicals and synthetic drugs. The in silicoanalysis of HE glycoprotein and these datasets revealed the robust natural repositories as well as the potency of current therapeutic drugs. This study found prominent interactions between the selected target and compounds. Among all datasets, Withanoside V, Racemoside C, Withanoside IV, Shatavarin VII and Shatavarin X emerged as promising compounds that targeted with the best binding affinity. Notably, the pharmacophore modeling of these compounds revealed high potency towards the inhibition of HE glycoprotein. The MD simulations were depicted as the best binding stability with correlative motions generated from the Dynamic cross-correlation matrix. Also, the distribution of 
hydrogen bonds and the energy contribution of all simulated complexes were calculated through binding free energy. Overall, these in silicoanalysis revealed that the coronavirus hemagglutinin-esterase glycoprotein complex was inhibited by Withania somnifera(ashwagandha) and Asparagus racemosus (shatavari). This is primary level anticipation which can be validated by in vitro andin vivo studies.

\section{CONFLICTS OF INTEREST}

The authors declare that there are no conflicts of interest.

\section{ACKNOWLEDGMENTS}

This work is supported by the Financial Assistance Programme - Department of Science and Technology [Grant number GSBTM/MD/JDR/1409/2017-18] and Gujarat Council on Science and Technology [GUJCOST/Supercomputer/2019-20/1359]. The authors gratefully acknowledge the Department of Botany, Bioinformatics and Climate Change Impacts Management, Gujarat University for providing an opportunity to access the bioinformatics research facilities.

\section{REFERENCES}

1. Pene F, Merlat A, Vabret A, et al. Coronavirus 229E-related pneumonia in immunocompromised patients. Clinical infectious diseases. 2003;37(7):929-932.

2. Lu R, Zhao X, Li J, et al. Genomic characterisation and epidemiology of 2019 novel coronavirus: implications for virus origins and receptor binding. The Lancet.2020;395(10224):565-574.

3. Zhou P, Yang X-L, Wang X-G, et al. A pneumonia outbreak associated with a new coronavirus of probable bat origin. nature. 2020;579(7798):270-273.

4. Belouzard S, Millet JK, Licitra BN, Whittaker GR. Mechanisms of coronavirus cell entry mediated by the viral spike protein. Viruses. 2012;4(6):1011-1033.

5. Tai W, Zhao G, Sun S, et al. A recombinant receptor-binding domain of MERS-CoV in trimeric form protects human dipeptidyl peptidase 4 (hDPP4) transgenic mice from MERS-CoV infection. Virology. 2016;499:375-382.

6. Tai W, He L, Zhang X, et al. Characterization of the receptor-binding domain (RBD) of 2019 novel coronavirus: implication for development of RBD protein as a viral attachment inhibitor and vaccine. Cellular 83 molecular immunology. 2020:1-8.

7. Belouzard S, Chu VC, Whittaker GR. Activation of the SARS coronavirus spike protein via sequential proteolytic cleavage at two distinct sites. Proceedings of the National Academy of Sciences. 2009;106(14):58715876 .

8. Infantino M, Damiani A, Gobbi FL, et al. Serological assays for SARS-CoV-2 infectious disease: benefits, limitations and perspectives. Isr Med Assoc J.2020;22(4):203-210.

9. Zeng Q, Langereis MA, van Vliet AL, Huizinga EG, de Groot RJ. Structure of coronavirus hemagglutininesterase offers insight into corona and influenza virus evolution. Proceedings of the National Academy of Sciences.2008;105(26):9065-9069.

10. Prajapat M, Sarma P, Shekhar N, et al. Drug targets for corona virus: A systematic review. Indian journal of pharmacology. 2020;52(1):56.

11. Rabaan AA, Al-Ahmed SH, Haque S, et al. SARS-CoV-2, SARS-CoV, and MERS-CoV: a comparative overview.Infez Med. 2020;28(2):174-184.

12. Fantini J, Di Scala C, Chahinian H, Yahi N. Structural and molecular modeling studies reveal a new mechanism of action of chloroquine and hydroxychloroquine against SARS-CoV-2 infection. International journal of antimicrobial agents. 2020:105960. 
13. Fang L, Karakiulakis G, Roth M. Are patients with hypertension and diabetes mellitus at increased risk for COVID-19 infection? The Lancet Respiratory Medicine.2020;8(4):e21.

14. Xu X, Chen P, Wang J, et al. Evolution of the novel coronavirus from the ongoing Wuhan outbreak and modeling of its spike protein for risk of human transmission.Science China Life Sciences. 2020;63(3):457-460.

15. Letko M, Marzi A, Munster V. Functional assessment of cell entry and receptor usage for SARS-CoV-2 and other lineage B betacoronaviruses. Nature microbiology.2020;5(4):562-569.

16. Holmes KV. SARS-associated coronavirus. New England Journal of Medicine.2003;348(20):1948-1951.

17. Patel CN, Chirag N, Pandya D, Himanshu A, Rawal D, Rakesh M. Identification of Potential Binders of the SARS-Cov-2 Spike Protein via Molecular Docking, Dynamics Simulation and Binding Free Energy Calculation. 2020.

18. Ashour HM, Elkhatib WF, Rahman M, Elshabrawy HA. Insights into the recent 2019 novel coronavirus (SARS-CoV-2) in light of past human coronavirus outbreaks.Pathogens. 2020;9(3):186.

19. Devaux CA, Rolain J-M, Colson P, Raoult D. New insights on the antiviral effects of chloroquine against coronavirus: what to expect for COVID-19? International journal of antimicrobial agents. 2020:105938.

20. Kumar A, Kumar R, Sharma M, Kumar U, Gajula M, Singh KP. Uttarakhand Medicinal Plants Database (UMPDB): A Platform for Exploring Genomic, Chemical, and Traditional Knowledge.Data. 2018;3(1):7.

21. Kumar A, Choudhir G, Shukla SK, et al. Identification of phytochemical inhibitors against main protease of COVID-19 using molecular modeling approaches. Journal of Biomolecular Structure and Dynamics. 2020(just-accepted):1-21.

22. Patel CN, Georrge JJ, Modi KM, et al. Pharmacophore-based virtual screening of catechol-omethyltransferase (COMT) inhibitors to combat Alzheimer's disease. Journal of Biomolecular Structure and Dynamics.2018;36(15):3938-3957.

23. Mwitari PG, Ayeka PA, Ondicho J, Matu EN, Bii CC. Antimicrobial activity and probable mechanisms of action of medicinal plants of Kenya: Withania somnifera, Warbugia ugandensis, Prunus africana and Plectrunthus barbatus. PloS one.2013;8(6).

24. Sabde S, Bodiwala HS, Karmase A, et al. Anti-HIV activity of Indian medicinal plants. Journal of natural medicines. 2011;65(3-4):662-669.

25. Lamari Z, Larbi R, Negache H. Trace element content of Zingiber officinalis and Salvia officinalis medicinal plants from Algeria. Journal of Radioanalytical and Nuclear Chemistry. 2016;309(1):17-22.

26. Balasubramanian G, Sarathi M, Kumar SR, Hameed AS. Screening the antiviral activity of Indian medicinal plants against white spot syndrome virus in shrimp.Aquaculture. 2007;263(1-4):15-19.

27. Dao TT, Nguyen PH, Won HK, et al. Curcuminoids from Curcuma longa and their inhibitory activities on influenza A neuraminidases. Food chemistry. 2012;134(1):21-28.

28. Ishnava KB, Chauhan K, Bhatt C. Screening of antifungal activity of various plant leaves extracts from Indian plants. Archives of phytopathology and plant protection.2012;45(2):152-160.

29. Kim S, Thiessen PA, Bolton EE, et al. PubChem substance and compound databases. Nucleic acids research. 2016;44(D1):D1202-D1213.

30. Krieger E, Darden T, Nabuurs SB, Finkelstein A, Vriend G. Making optimal use of empirical energy functions: force-field parameterization in crystal space.Proteins: Structure, Function, and Bioinformatics.2004;57(4):678-683.

31. Kadukova M, Chupin V, Grudinin S. Docking rigid macrocycles using Convex-PL, AutoDock Vina, and RDKit in the D3R Grand Challenge 4. Journal of Computer-Aided Molecular Design. 2020;34(2):191-200. 
32. Ren J, Yuan X, Li J, et al. Assessing the performance of the g_mmpbsa tools to simulate the inhibition of oseltamivir to influenza virus neuraminidase by molecular mechanics Poisson-Boltzmann surface area methods. Journal of the Chinese Chemical Society. 2020;67(1):46-53.

33. Mabbitt PD, Correy GJ, Meirelles T, Fraser NJ, Coote ML, Jackson CJ. Conformational disorganization within the active site of a recently evolved organophosphate hydrolase limits its catalytic efficiency. Biochemistry.2016;55(9):1408-1417.

34. Yadav DK, Kumar S, Teli MK, Kim MH. Ligand-based pharmacophore modeling and docking studies on vitamin D receptor inhibitors. Journal of Cellular Biochemistry. 2020.

35. Kumar SP, Patel CN, Jha PC, Pandya HA. Molecular dynamics-assisted pharmacophore modeling of caspase-3-isatin sulfonamide complex: Recognizing essential intermolecular contacts and features of sulfonamide inhibitor class for caspase-3 binding. Computational biology and chemistry.2017;71:117-128.

36. Mohan A, Kirubakaran R, Parray J, Sivakumar R, Murugesh E, Govarthanan M. Ligand-based pharmacophore filtering, atom based 3D-QSAR, virtual screening and ADME studies for the discovery of potential ck2 inhibitors. Journal of Molecular Structure. 2020;1205:127670.

37. Lipinski CA, Lombardo F, Dominy BW, Feeney PJ. Experimental and computational approaches to estimate solubility and permeability in drug discovery and development settings.Advanced drug delivery reviews. 1997;23(1-3):3-25.

38. Veber DF, Johnson SR, Cheng H-Y, Smith BR, Ward KW, Kopple KD. Molecular properties that influence the oral bioavailability of drug candidates. Journal of medicinal chemistry. 2002;45(12):2615-2623.

39. Naidoo D, Roy A, Slavětínská LP, Chukwujekwu J, Gupta S, Van Staden J. New role for crinamine as a potent, safe and selective inhibitor of human monoamine oxidase B: In vitro and in silico pharmacology and modeling. Journal of ethnopharmacology. 2020;248:112305.

40. Daina A, Michielin O, Zoete V. SwissADME: a free web tool to evaluate pharmacokinetics, drug-likeness and medicinal chemistry friendliness of small molecules.Scientific reports. 2017;7:42717.

41. Singh VK, Srivastava R, Gupta PSS, et al. Anti-HIV potential of diarylpyrimidine derivatives as nonnucleoside reverse transcriptase inhibitors: Design, synthesis, docking, TOPKAT analysis and molecular dynamics simulations. Journal of Biomolecular Structure and Dynamics. 2020:1-17.

42. Krieger E, Nielsen JE, Spronk CA, Vriend G. Fast empirical pKa prediction by Ewald summation.Journal of molecular graphics and modelling. 2006;25(4):481-486.

43. Jakalian A, Jack DB, Bayly CI. Fast, efficient generation of high-quality atomic charges. AM1-BCC model: II. Parameterization and validation. Journal of computational chemistry. 2002;23(16):1623-1641.

44. Berendsen HJ, Postma Jv, van Gunsteren WF, DiNola A, Haak JR. Molecular dynamics with coupling to an external bath. The Journal of chemical physics.1984;81(8):3684-3690.

45. Mark P, Nilsson L. Structure and dynamics of the TIP3P, SPC, and SPC/E water models at 298 K. The Journal of Physical Chemistry A. 2001;105(43):9954-9960.

46. Kumar SP, Patel CN, Rawal RM, Pandya HA. Energetic contributions of amino acid residues and its cross-talk to delineate ligand-binding mechanism. Proteins: Structure, Function, and Bioinformatics. 2020.

47. Gould J. Morpheus: Versatile matrix visualization and analysis software [WWW Document]. URL https://software broadinstitute org/morpheus/(accessed 91 18). 2016.

48. Wang W, Kollman PA. Free energy calculations on dimer stability of the HIV protease using molecular dynamics and a continuum solvent model. Journal of molecular biology. 2000;303(4):567-582.

49. Wang W, Kollman PA. Computational study of protein specificity: the molecular basis of HIV-1 protease drug resistance. Proceedings of the National Academy of Sciences.2001;98(26):14937-14942. 
50. Su J, Liu X, Zhang S, Yan F, Zhang Q, Chen J. A computational insight into binding modes of inhibitors XD29, XD35, and XD28 to bromodomain-containing protein 4 based on molecular dynamics simulations. Journal of Biomolecular Structure and Dynamics. 2018;36(5):1212-1224.

51. Wang C, Greene D, Xiao L. Recent developments and applications of the MMPBSA method. Front Mol Biosci. In:2018.

52. Razzaghi-Asl N, Mirzayi S, Mahnam K, Sepehri S. Identification of COX-2 inhibitors via structurebased virtual screening and molecular dynamics simulation. Journal of Molecular Graphics and Modelling. 2018;83:138-152.

\section{Author contributions}

C.N.P. and H.A.P. conceptualized and designed the project. C.N.P. and D.P.P. developed methodology. C.N.P., D.G.J. and S.P.J. acquired data. C.N.P., D.G.J., S.P.J. and D.P.P. analyzed and interpreted data. C.N.P., S.P.J., D.G.J., F.J.G. and H.A.P. wrote manuscript. D.P.P. and F.J.G. provided technical support and H.A.P. supervised study. H.A.P. acquired grant. The whole manuscript was approved by all authors.

\section{Figure Legends}

Figure-1 (A) Docked pose of the HE glycoprotein with re-docked ligand 4,9-O-diacetyl sialic acid (Green color - native ligand and Yellow color - amino acids) and (B) Protein-ligand interaction maps developed from PoseView.

Figure-2 Docked pose of the HE glycoprotein with top-ranked plant-derived phytochemicals (Green color - ligands and Cyan color - amino acids). (A) Withanoside V, (B) Racemoside C, (C) Withanoside IV, (D) Shatavarin VII and (E) Shatavarin X.

Figure-3 Docked pose of the HE glycoprotein with top-ranked Synthetic drugss (Pink color - ligands and White color - amino acids). (A) Remdesivir, (B) Saquinavir, (C) Delavirdine, (D) Indinavir and (E) Fosamprenavir.

Figure-4 Ligand-based pharmacophore modelling: (A) Mapped pharmacophore features of co-crystal ligand, (B) Comparative pharmacophore features of co-crystal ligand and top-ranked phytochemicals, (C) Comparative pharmacophore features of co-crystal ligand and top-ranked protease inhibitors (Hydrogen Bond Donor: Pink sphere, Hydrogen Bonds Acceptor: Green Sphere and Ionizable area: Blue, Orange and Red Positive Aromatic rings).

Figure-5 Molecular Dynamics Simulation analysis: Time vs. Total energy and Time vs. RMSD graph. Color denotation: Yellow-Withanoside V; Purple-Racemoside C; Green-Withanoside IV; Magenta-Shatavarin VII; Skyblue-Shatavarin X.

Figure-6 Structural Stability analysis: Pocket residue vs. Pocket RMSD and Pocket residues vs. Pocket RMSF. Color denotation: Yellow-Withanoside V; Purple-Racemoside C; Green-Withanoside IV; MagentaShatavarin VII; Skyblue-Shatavarin X.

Figure-7 Graphical representation of the interactions between Coronavirus Hemagglutinin-Esterase with Withanoside $\mathrm{V}$ simulated molecule by Discovery studio visualiser ((A) 0 nanoseconds, (B) 5 nanoseconds, (C) 10 nanoseconds, (D) 15 nanoseconds, (E) 20 nanoseconds).

Figure-8 Graphical representation of the interactions between Coronavirus Hemagglutinin-Esterase with Racemoside C simulated molecule by Discovery studio visualiser ((A) 0 nanoseconds, (B) 5 nanoseconds, (C) 10 nanoseconds, (D) 15 nanoseconds, (E) 20 nanoseconds).

Figure-9 Graphical representation of the interactions between Coronavirus Hemagglutinin-Esterase with Withanoside IV simulated molecule by Discovery studio visualiser ((A) 0 nanoseconds, (B) 5 nanoseconds, (C) 10 nanoseconds, (D) 15 nanoseconds, (E) 20 nanoseconds). 
Figure-10 Graphical representation of the interactions between Coronavirus Hemagglutinin-Esterase with Shatavarin VII simulated molecule by Discovery studio visualiser ((A) 0 nanoseconds, (B) 5 nanoseconds, (C) 10 nanoseconds, (D) 15 nanoseconds, (E) 20 nanoseconds).

Figure-11 Graphical representation of the interactions between Coronavirus Hemagglutinin-Esterase with Shatavarin X simulated molecule by Discovery studio visualiser ((A) 0 nanoseconds, (B) 5 nanoseconds, (C) 10 nanoseconds, (D) 15 nanoseconds, (E) 20 nanoseconds).

Figure-12 Binding free energy calculations of coronavirus hemagglutinin-esterase and top ranked phytochemicals. Color denotation: Yellow-Withanoside V; Purple-Racemoside C; Green-Withanoside IV; Magenta-Shatavarin VII; Skyblue-Shatavarin X.

\section{Table Legends}

Table-1 Binding modes of top-ranked plant-derived phytochemicals with HE glycoprotein with Binding energy, Hydrogen bonds, Dissociation constant, Efficiency and contacting receptor residues.

Table-2 Binding modes of top-ranked Synthetic drugss with HE glycoprotein with Binding energy, Hydrogen bonds, Dissociation constant, Efficiency and contacting receptor residues.

Table-3 Prediction of Lipinski's 'Rule of 5', Veber and ADMET for the top-ranked phytochemicals.

Table-4 Prediction of Lipinski's 'Rule of 5', Veber and ADMET for the top-ranked FDA-approved Protease inhibitors.

Table-5 The Binding free energy of binding for a series of top-ranked hits using MM-/PBSA approach.
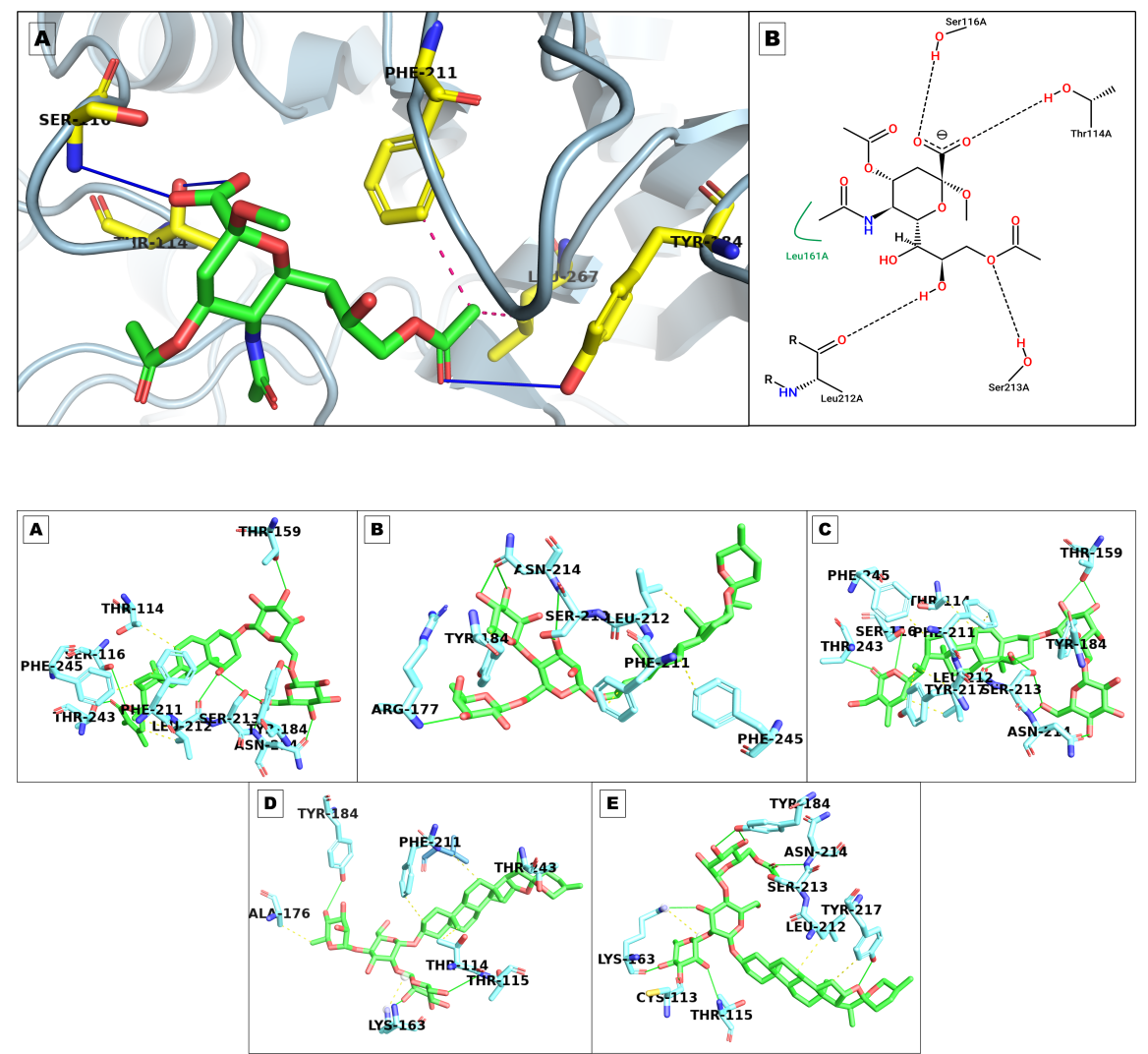

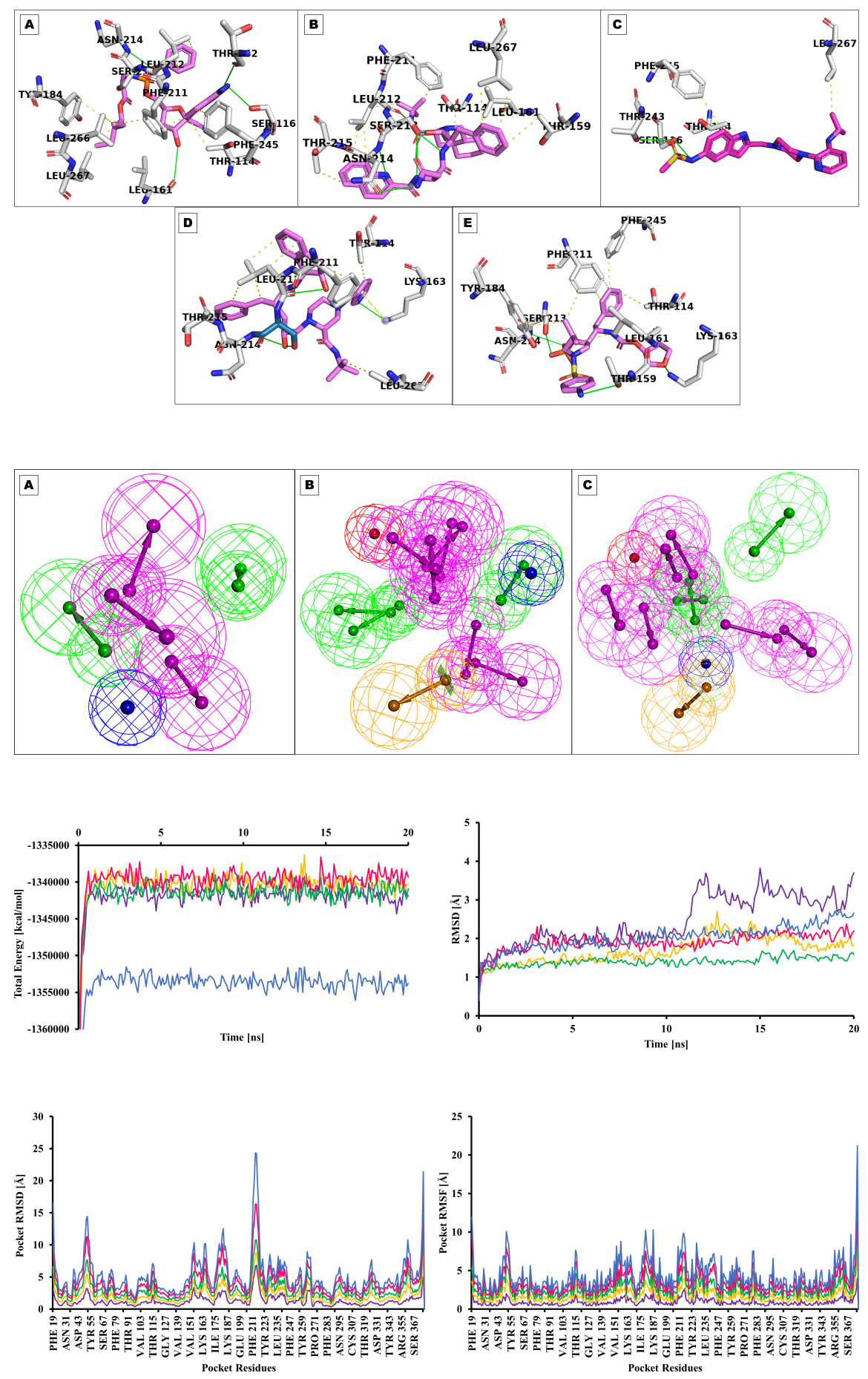

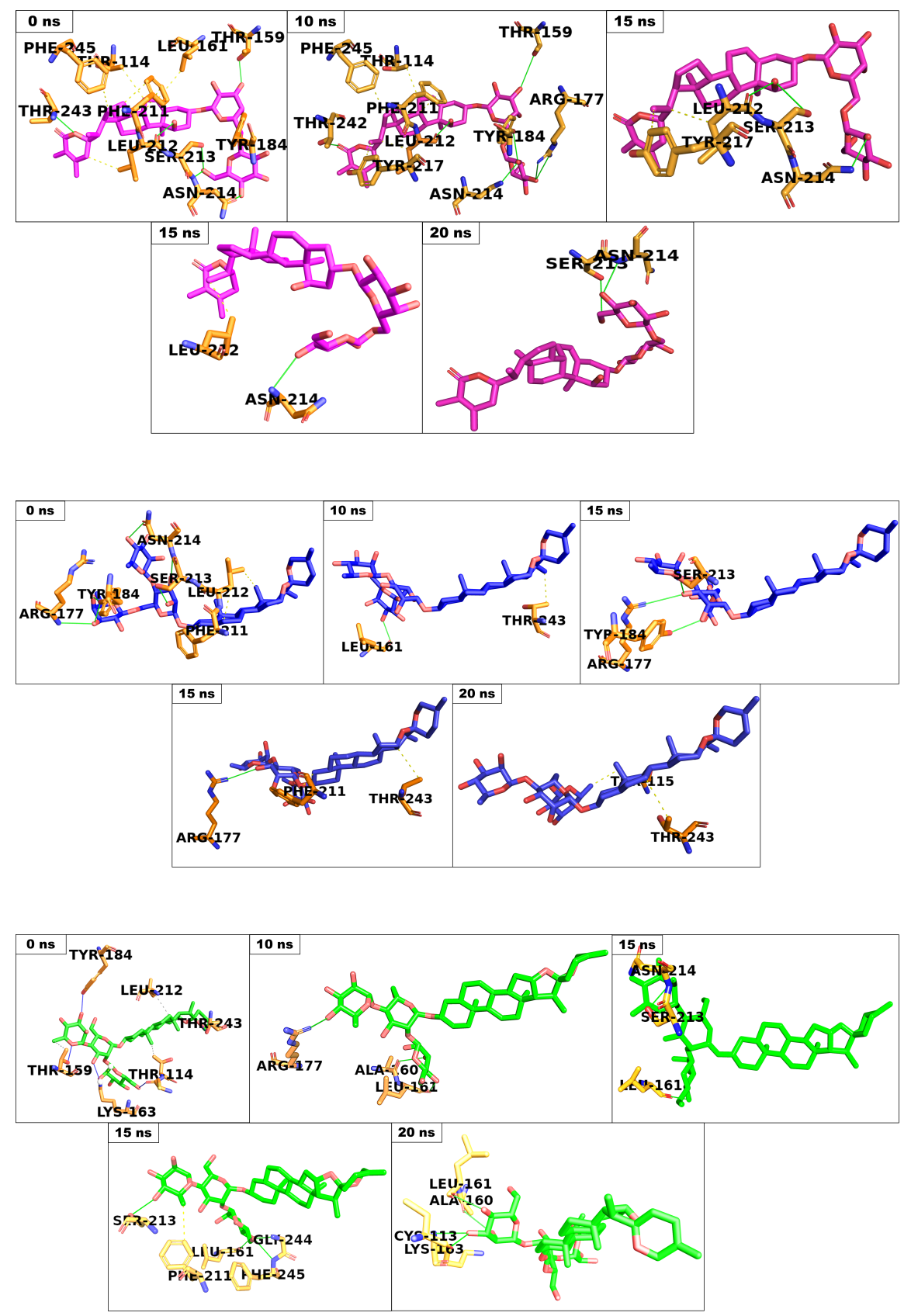

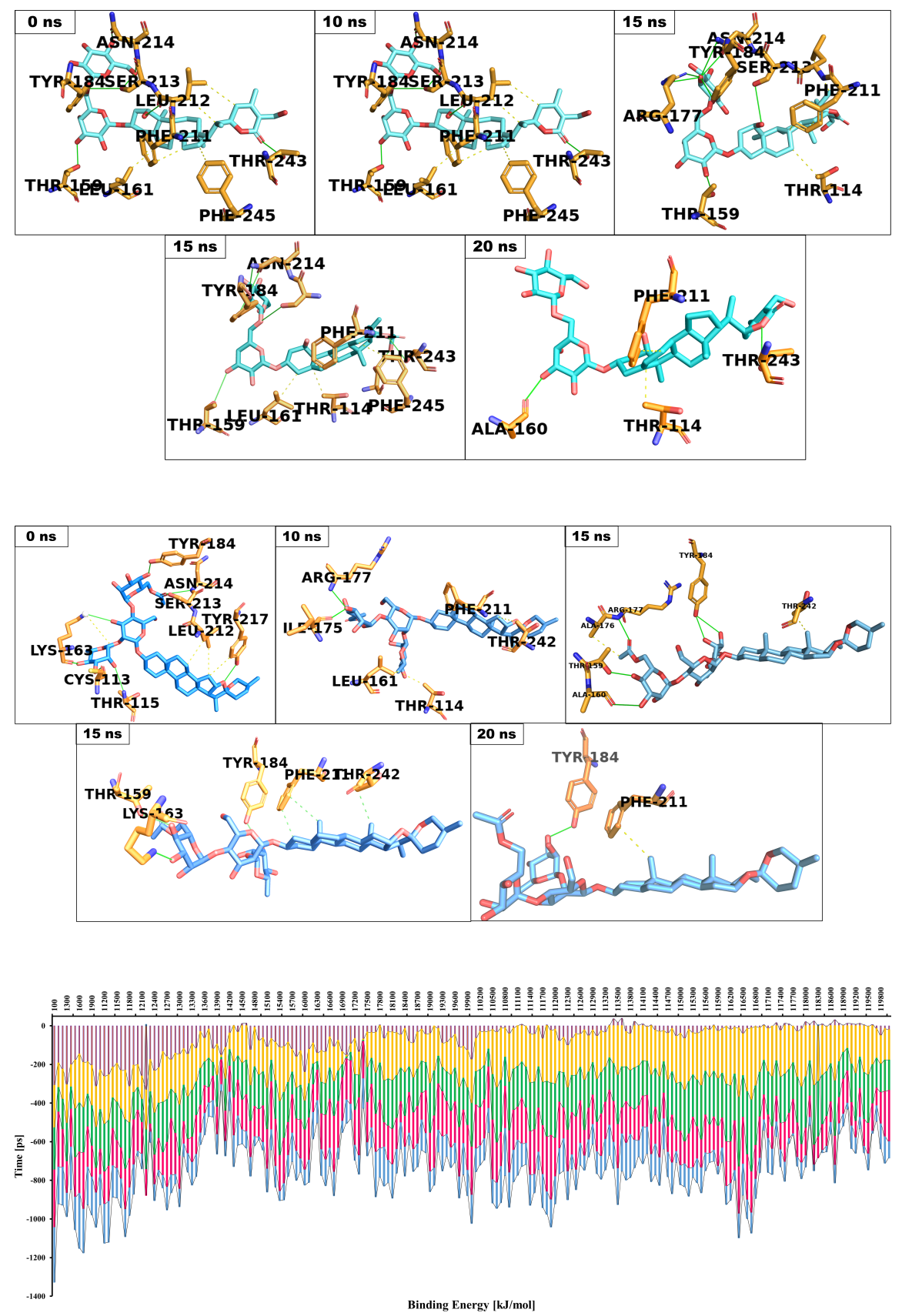

\section{Hosted file}

Table-1.docx available at https://authorea.com/users/334216/articles/460266-molecularinteraction-studies-of-natural-compounds-against-coronavirus-hemagglutinin-esterase-incomplex-with-ace2-receptor-an-in-silico-approach

Hosted file

Table-2.docx available at https://authorea.com/users/334216/articles/460266-molecularinteraction-studies-of-natural-compounds-against-coronavirus-hemagglutinin-esterase-incomplex-with-ace2-receptor-an-in-silico-approach 


\section{Hosted file}

Table-3.docx available at https://authorea.com/users/334216/articles/460266-molecularinteraction-studies-of-natural-compounds-against-coronavirus-hemagglutinin-esterase-incomplex-with-ace2-receptor-an-in-silico-approach

\section{Hosted file}

Table-4.docx available at https://authorea.com/users/334216/articles/460266-molecularinteraction-studies-of-natural-compounds-against-coronavirus-hemagglutinin-esterase-incomplex-with-ace2-receptor-an-in-silico-approach 